\title{
Effect of selective vagal nerve stimulation on blood pressure, heart rate and respiratory rate in rats under metoprolol medication
}

\author{
Mortimer Gierthmuehlen ${ }^{1}$ and Dennis TT Plachta ${ }^{1,2}$
}

Selective vagal nerve stimulation (sVNS) has been shown to reduce blood pressure without major side effects in rats. This technology might be the key to non-medical antihypertensive treatment in patients with therapy-resistant hypertension. $\boldsymbol{\beta}$-blockers are the first-line therapy of hypertension and have in general a bradycardic effect. As VNS itself can also promote bradycardia, it was the aim of this study to investigate the influence of the $\beta 1$-selective blocker Metoprolol on the effect of sVNS especially with respect to the heart rate. In 10 male Wistar rats, a polyimide multichannel-cuff electrode was placed around the vagal nerve bundle to selectively stimulate the aortic depressor nerve fibers. The stimulation parameters were adapted to the thresholds of individual animals and were in the following ranges: frequency $30-50 \mathrm{~Hz}$, amplitude $0.3-1.8 \mathrm{~mA}$ and pulse width 0.3-1.3 ms. Blood pressure responses were detected with a microtip transducer in the carotid artery, and electrocardiography was recorded with s.c. chest electrodes. After IV administration of Metoprolol ( $2 \mathrm{mg} \mathrm{kg}^{-1}$ body weight), the animals' mean arterial blood pressure (MAP) and heart rate (HR) decreased significantly. Although the selective electrical stimulation of the baroreceptive fibers reduced MAP and HR, both effects were significantly alleviated by Metoprolol. As a side effect, the rate of stimulation-induced apnea significantly increased after Metoprolol administration. sVNS can lower the MAP under Metoprolol without causing severe bradycardia.

Hypertension Research (2016) 39, 79-87; doi:10.1038/hr.2015.122; published online 19 November 2015

Keywords: aortic depressor nerve; baroreflex; $\beta$-blocker; hypertension; resistant

\section{INTRODUCTION}

Arterial hypertension is a common disease with a prevalence of $30 \%{ }^{1}$ and is responsible for over $50 \%$ of cardio and cerebrovascular accidents. $^{2}$ Despite modern medical therapy, up to $30 \%$ of patients with arterial hypertension, a condition in which mental stress and the associated activation of the autonomic nervous system also have a significant role, ${ }^{3}$ cannot be treated sufficiently ${ }^{4}$ and do not reach a target blood pressure (BP) below $140 \mathrm{~mm} \mathrm{Hg}$. Knowledge of the reason of true and pseudo-resistance is scarce. ${ }^{5}$ Among other non-pharmaceutical treatments, the electrical activation of the arterial baroreceptors at the carotid bifurcation has been investigated as a potential antihypertensive therapy. A new pacing device has been introduced to activate the baroreflex through bilateral carotid sinus stimulation. ${ }^{6,7}$ This system, called Rheos, was able to continuously reduce the arterial BP by up to $35 \mathrm{~mm} \mathrm{Hg}$ over a 12-month period, but yielded a periprocedural complication rate of $\sim 20 \%{ }^{8}$

Arterial baroreceptors are also located in the aortic arch (Figure 1). These baroreceptors send their information to the nucleus tractus solitarii in the brainstem by means of specialized and dedicated fibers of the vagal nerve. 9 The electrical stimulation of these surgically isolated fibers, called aortic depressor nerves $(\mathrm{ADN})$, if present as a separate strand outside the vagal nerve in rats, also reduces the $\mathrm{BP}$ through activation of the baroreflex. ${ }^{10}$ As vagal nerve stimulation (VNS) is already being used as a treatment for intractable epilepsy and is associated with a very low complication rate, ${ }^{11,12}$ we have recently proposed a technique of selective VNS (sVNS) with a multichannel cuff electrode (MCE) to activate the baroreflex. ${ }^{13}$ In this approach, the MCE is wrapped around the vagal nerve bundle, including the vagus, the ADN, the laryngeal recurrent nerve, small supply vessels and some connective and fat tissue. A signal detection algorithm, which is based on coherent averaging, was used to locate the baroreceptive fibers inside the vagal nerve bundle and identify the appropriate electrode to selectively trigger the baroreflex. Recently, we were able to demonstrate the reduction in arterial BP with minimal side effects during sVNS in male Wistar rats with no medical treatment. ${ }^{14}$

Antihypertensive drugs have a benefitial effect on the cardiovascular system well beyond the pure reduction of BP. ${ }^{15}$ In humans, $\beta$-blockers are among the first-line treatment options for arterial hypertension, and even if sVNS will become a treatment option of resistant hypertension in future, $\beta$-blockers will probably be continued owing

${ }^{1}$ Department of Neurosurgery, University Medical Center Freiburg, Freiburg, Germany and ${ }^{2}$ Department of Microsystem Engineering (IMTEK), Laboratory for Biomedical Microtechnology, Freiburg, Germany

Correspondence: Dr M Gierthmuehlen, Department of Neurosurgery, University Medical Center Freiburg, Breisacher Str. 64, Freiburg 79106, Germany.

E-mail: mortimer.gierthmuehlen@uniklinik-freiburg.de

Received 12 May 2015; revised 6 September 2015; accepted 13 September 2015; published online 19 November 2015 


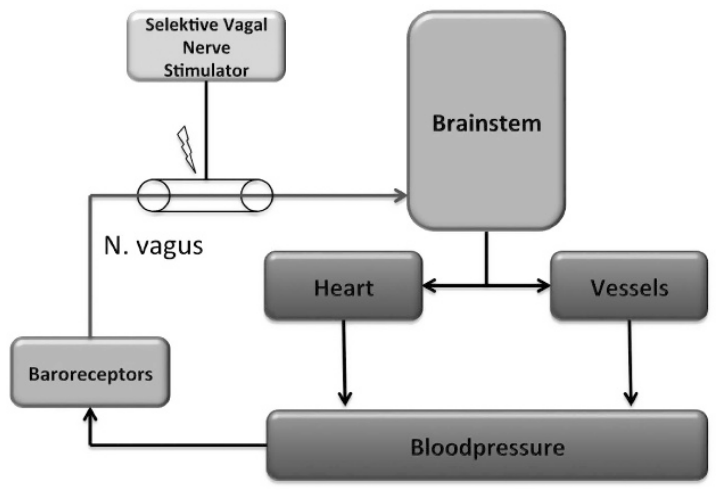

Figure 1 Basic concept of the closed loop of the baroreflex. The selective vagal nerve stimulator overwrites the signals deriving from the aortic baroreceptors and activates the baroreflex in order to lower the blood pressure. A full color version of this figure is available at Hypertension Research online.

to their benefitial influence, for example, on cardiac efficacy. As both treatment- $\beta$-blockers and sVNS - have the potential to promote bradycardia, it is the aim of this study to investigate the influence of the $\beta 1$-selective agent Metoprolol on heart rate (HR), respiration rate (RR), BP and MAP during sVNS.

\section{METHODS}

The study was approved by the Regierungspraesidium Freiburg, Baden-Wuerttemberg and the Animal Committee of the University of Freiburg (G13/44). The 'Principles of laboratory animal care' (NIH publication no. 86-53 23, revised 1996) were followed for all experiments. The detailed system setup and signal analysis was previously described. ${ }^{14,16}$

\section{MCE and pressure transducer.}

The MCE used in the experiments featured 24 working electrodes arranged in 8 tripoles around the cuff perimeter $\left(45^{\circ}\right.$ spacing) and two large, ring-shaped counter electrodes. ${ }^{17}$ The signals of all 24 channels (arranged in 8 tripoles) were used to record the signals of the vagal nerve bundle. Two electrodes facing outside were used as reference electrodes. The total length of the cuff was $10 \mathrm{~mm}$, the inner diameter was $0.8 \mathrm{~mm}$ and the distance between the crosssectional electrodes was $2 \mathrm{~mm}$. The electrode sites and interconnection lines were sandwiched between two layers of polyimide (total thickness $11 \mu \mathrm{m}$ ). The thin film metallization of $300 \mathrm{~nm}$ sputtered platinum was coated with $1000 \mathrm{~nm}$ iridium oxide on the electrode sites. Three needle electrodes were placed subcutaneously on the right and left chest and on the left foot for ECG recording. We used a 1.5 French microtip transducer (Millar Instruments, Houston, TX, USA) for invasive BP monitoring. The transducer was connected to an in-house made amplifier and then put on the analog input of the dataacquisition system. ECG and BP were recorded with the same settings on separate input channels of the setup and RR was calculated from the cycles in the MAP.

\section{Surgical procedure}

Ten male Wistar rats (body weight 380-420 g, mean $382 \mathrm{~g}$ ) were anesthetized with 2-4 vol\% Isoflurane (Abbott, North Chicago, IL, USA) and received Carprofene (Rimadyl, Pfizer, New York City, NY, USA $-5 \mathrm{mg} \mathrm{kg}^{-1}$ body weight s.c.) for analgesia. Anesthesia was maintained with 1-2 vol\% Isoflurane regulated by the RR. The rats were placed on a regulated, electrically isolated heat pad (Harvard Apparatus, Holliston, MA, USA) and an IV catheter was placed in the tail-vein. Saline solution was provided at $1 \mathrm{ml} / 100 \mathrm{~g}$ body weight per hour. Through a ventral neck incision, the left neurovascular sheath was exposed and gently opened under a surgical microscope (VM900, MoellerWedel GmbH, Wedel, Germany). The carotid artery was ligated distally and temporarily interrupted proximally with an aneurysm-clip. Through a small incision, a 1.5 French microtip transducer (Millar Instruments) was inserted into the carotid artery and advanced until the Waynforth-position was reached. ${ }^{18}$ The MCE was gently wrapped around the vagal nerve bundle and connected to the data-acquisition system. The left side was used as this is anatomically the vagal nerve that innervates the aortic baroreceptors and which is used in almost all baroreflex experiments in the literature.

\section{Data acquisition and localization of the baroreceptive fibers}

The recording of neurograms and ECG was carried out with a PZ3 system attached to an RZ2 module (both Tucker-Davis Technologies, Alachua, FL, USA). The RZ2 was connected to a PC via a PCIe interface card. The PZ3 pre-amplifier recorded monopolar signals from each of the 24 electrodes and the 2 reference electrodes with a sampling rate of $12 \mathrm{kHz}$. All input signals were notch filtered $(50 \mathrm{~Hz})$. The recorded and digitized signals were band-pass filtered using Matlab (MathWorks, Natick, MA, USA; Butterworth 2nd order, $20-200 \mathrm{~Hz}$ ). The whole surgery took place in an in-house made metal Faraday cage made of copper wire connected to a common ground with all other electrical devices. As previously described, ${ }^{16}$ the tripole contacts located near the baroreceptive fibers of the ADN were identified through coherent averaging during baseline recording for $20 \mathrm{~min}$, and those contacts were chosen for the stimulation procedure described below.

\section{sVNS and drug administration}

Stimulation was done unilaterally with current controlled, charge-balanced biphasic rectangular pulses, which were generated in the RZ2 module, and fed into an in-house made 8-channel voltage-to-current-converter. The digitalanalog conversion rate was $24 \mathrm{kHz}$ with a resolution of 16-bit. The center electrode of the chosen tripole served as the cathode, and the two large peripheral ring electrodes were the anodes. After localization of the tripole(s) with BP-related activity, we proceeded with a low charge and short pulse width $(0.3 \mathrm{~mA}$ and $0.3 \mathrm{~ms}$ at $40 \mathrm{~Hz})$ stimulation to test the responsiveness of the stimulation site. In 8 out of 10 test animals, this level of stimulation already resulted in a detectable BP decrease of at least $2 \%$. We then continued with a stimulation pattern, which was applied in all test animals (amplitudes from 0.3 to $1.8 \mathrm{~mA}$, pulse widths of $0.3-1.3 \mathrm{~ms}, 0.1$ increments) with repetition rates of 30,40 and $50 \mathrm{~Hz}$. In two test animals, we had to increase the stimulation parameters to see a BP decrease and therefore started our stimulation pattern from these higher values $(0.6 \mathrm{~mA}$ and $0.7 \mathrm{~ms})$. In three animals, we conducted additional stimulation below and beyond the borders of our fixed stimulation pattern to validate the responsiveness of the stimulation at very high and very low levels (durations of 0.1 and $0.2 \mathrm{~ms}$, as well as $1.4-1.8 \mathrm{~ms}$ ). Each combination of stimulation parameters was repeated three times in an arbitrary order. All stimulations contained 100 pulses, which resulted in sweep durations of $3.3 \mathrm{~s}$ for $30 \mathrm{~Hz}, 2.5 \mathrm{~s}$ for $40 \mathrm{~Hz}$ and $2 \mathrm{~s}$ for $50 \mathrm{~Hz}$. The intersweep intervall was at least $10 \mathrm{~s}$. Different stimulation parameters were separated by a break of at least $20 \mathrm{~s}$.

After finishing the initial recording and stimulation of the control data, $2 \mathrm{mg} \mathrm{kg}^{-1}$ body weight Metoprolol (Beloc, AstraZeneca, Wedel, Germany) was intravenously administered over $5 \mathrm{~min}$. The rats were allowed to adapt to this medication for another $5 \mathrm{~min}$. For comparative reasons, the stimulation pattern that was used before the application of Metoprolol was then repeated, independent of whether we saw a BP decrease at a given parameter combination. After the experiment and still under deep anesthesia the rats we perfused with saline and paraformaldehyde and the vagal nerve was resected.

\section{Statistics and data analysis}

The stimulation-related BP reductions were normalized to the percent of the original MAP. Because the pressure amplitude decreased with Metoprolol, this step was necessary to keep the BP results of the pre- and post-Metoprolol scenario comparable. The multivariate variables were pooled across all 10 test animals. For statistical analysis, we built bins of stimulation parameters for the stimulation amplitude and for the stimulation pulse width. These bins were then tested for normality using the D'Agostini Omnibus normality test, as well as the Shapiro-Wilk test. This procedure was performed independently for the MAP-decrease pre- and post-Metoprolol and for HR. The Kruskal-Wallis test and the Dunn's test for multiple comparisons were chosen to compare the different bins. The PQ-intervals were extracted from the ECG recording by 


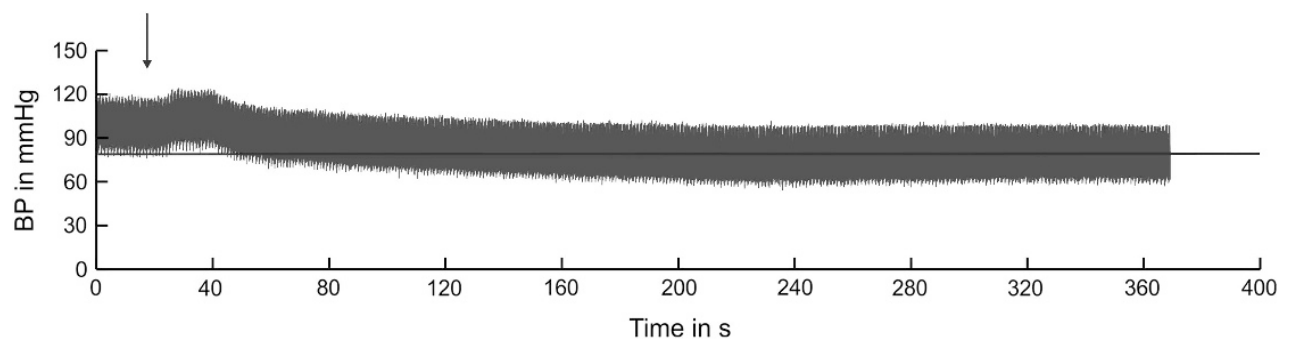

Figure 2 After the application of Metoprolol (arrow), the BP decreases until a steady state is reached ( 200s after injection). A full color version of this figure is available at Hypertension Research online.
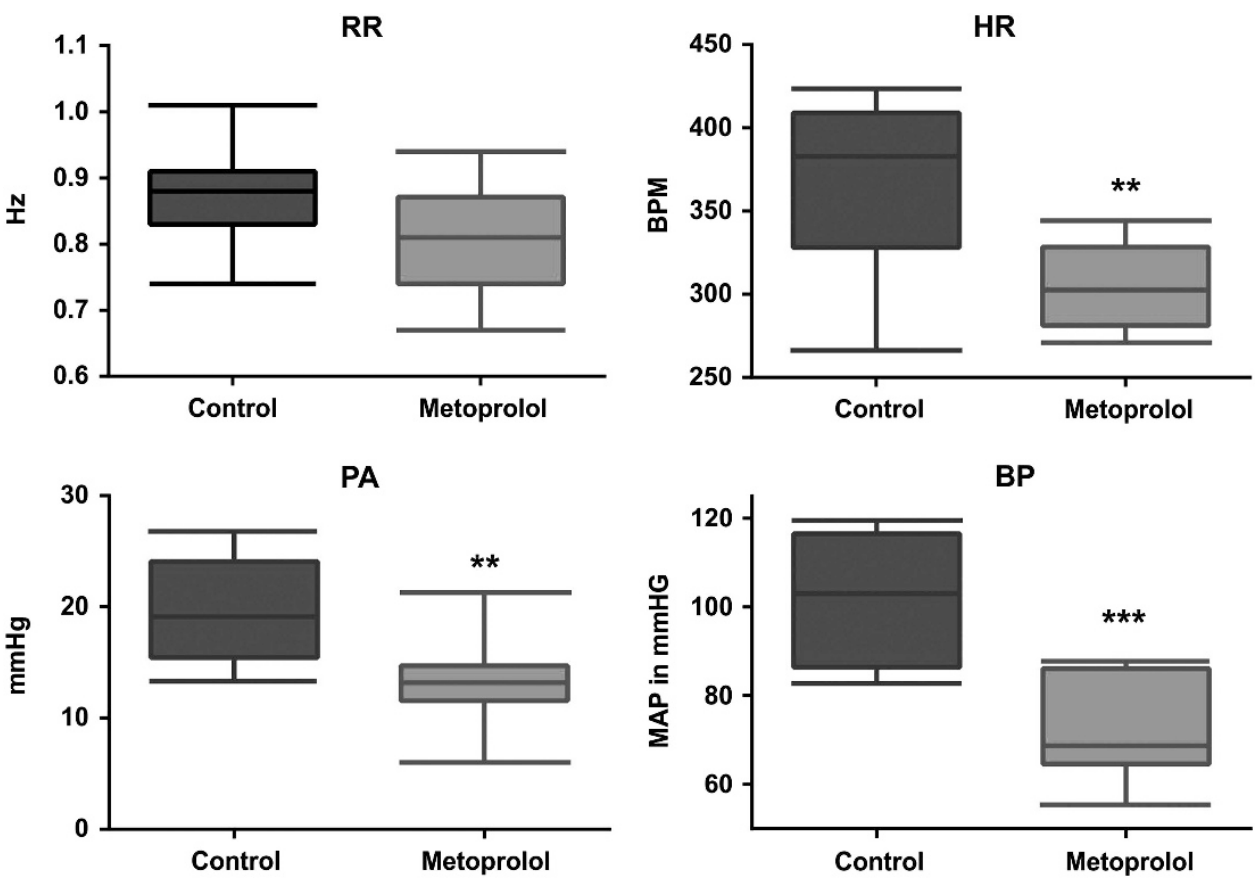

Figure 3 The effect of Metoprolol on respiration rate (RR), heart rate (HR), pulse amplitude (PA) and mean arterial blood pressure (MAP). The effects were significant for MAP $(P=0.0002), \mathrm{PA}(P=0.006)$ and $\mathrm{HR}(P=0.003)$, but not significant on $\mathrm{RR}(P=0.0575)$. A full color version of this figure is available at Hypertension Research online.

means of a in-house-written Matlab script. Some weaker ECG signals $(n=3)$ were enhanced using Wiener Filter. The stimulation currents interrupted the recording of the ECG signals. We therefore assessed the PQ-intervals of each animal from four different time intervals (each at least $5 \mathrm{~min}$ ), which did not contain any stimulation. The intervals were (i) before stimulation and drug administration, (ii) immediately after selective stimulation, (iii) during application of Metoprolol and (iv) after stimulation under Metoprolol condition. The PQ-intervals of these intervals were pooled, respectively, and compared using the Kruskal-Wallis test and the Dunn's Test for multiple comparisons.

All box plots of Figures 3 and 5 show the mean value, with the two inner quartiles as the box frame. The whiskers present the minimum and maximum. Significance level stars were used with the following convention: ${ }^{*} P=0.05-0.01$, ${ }^{* *} P<0.01-0.001,{ }^{* * *} P<0.001-0.0001,{ }^{* * * *} P<0.0001$.

\section{RESULTS}

Baselines readings for $\mathrm{HR}, \mathrm{RR}$, pulse amplitude and MAP before and after administration of Metoprolol

On average $(n=10)$, it took 214.7 s (s.d. $\pm 85.22 \mathrm{~s})$ until the BP reached a steady level after Metoprolol application (Figure 2). For the comparison of control vs Metoprolol, we recorded the vital parameters of all 10 animals at the beginning of the experiment (before stimulation and application of Metoprolol) and after steady state was established (before stimulation), for $10 \mathrm{~min}$. The results from all 10 animals were pooled and tested for normality using the Shapiro-Wilk test. After acceptance of normality we compared the control and Metoprolol data for each vital parameter with a two-tailed unpaired Welch $t$-test. (Figure 3 ). Metoprolol significantly $(P=0.0002)$ reduced MAP from $101.3 \mathrm{~mm} \mathrm{Hg}( \pm 14.7 \mathrm{~mm} \mathrm{Hg}$, s.d. $)$ to $72.7 \mathrm{~mm} \mathrm{Hg}( \pm 11.6 \mathrm{~mm} \mathrm{Hg}$, s.d. $)$ and pressure amplitude changed significantly $(P=0.006)$ from $19.7 \mathrm{~mm} \mathrm{Hg}( \pm 4.9 \mathrm{~mm} \mathrm{Hg}$, s.d. $)$ to $13.3 \mathrm{~mm} \mathrm{Hg}( \pm 4.0 \mathrm{~mm} \mathrm{Hg}$, s.d.). The HR was also significantly $(P=0.003)$ influenced by Metoprolol and decreased from 369.9 b.p.m. ( \pm 49.66 b.p.m., s.d.) to 304.6 b.p.m. ( \pm 26.6 b.p.m., s.d.). The RR was reduced from $0.88 \mathrm{~Hz}( \pm 0.08 \mathrm{~Hz}$, s.d.) to $0.8 \mathrm{~Hz}$ $( \pm 0.085 \mathrm{~Hz}$, s.d.), but this change was not significant $(P=0.0575)$.

Response of MAP to electrical stimulation before and after administration of Metoprolol

Metoprolol reduced BP, HR and pressure amplitude significantly, but selective stimulation still triggered a BP decrease at higher stimulation intensities (Figure 4). Except for the lowest amplitude and stimulation pulse width bins, the BP decrease of the control was significantly higher than the BP decrease under Metoprolol (Figure 5, first row). 


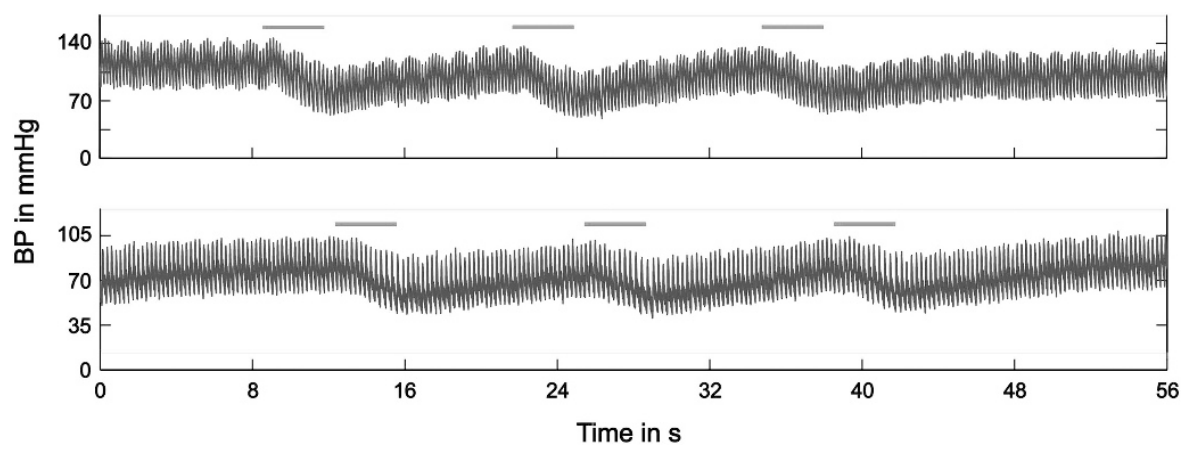

Figure 4 An example for the effect of sVNS on the BP before (upper row) und after (lower row) administration of Metoprolol ( $2 \mathrm{mg} \mathrm{kg}^{-1}$ body weight). Although HR and BP decreased, the stimulation still showed a BP-lowering effect. A full color version of this figure is available at Hypertension Research online.

Control
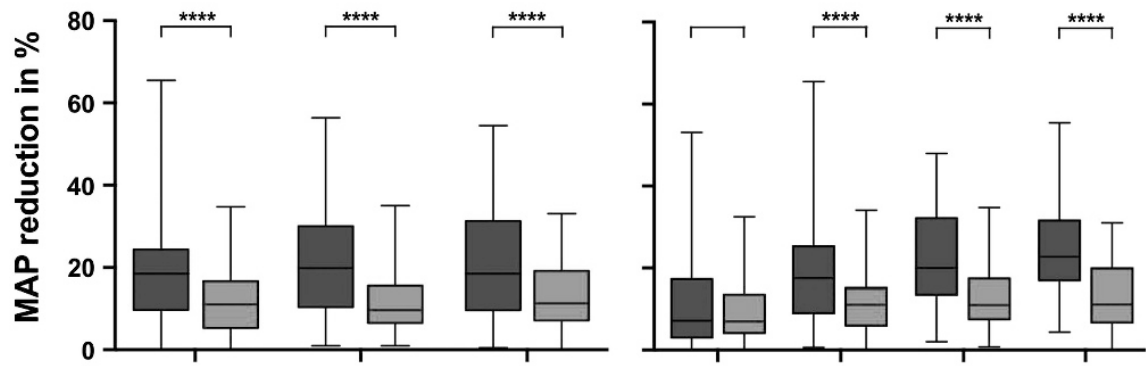

Metoprolol
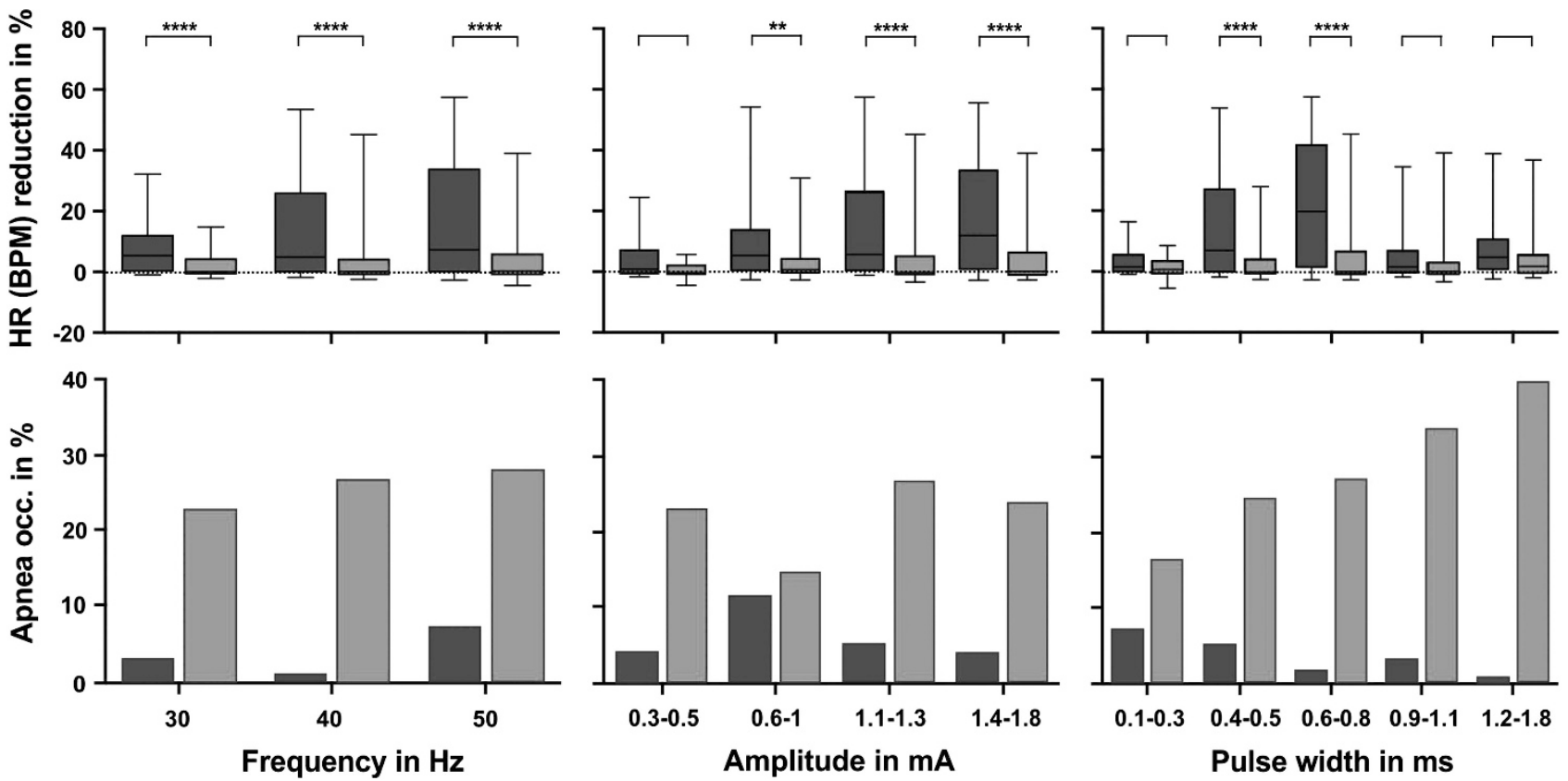

Figure 5 Effect of selective vagal nerve stimulation (sVNS) blood pressure (BP; mean arterial blood pressure (MAP) decrease in percentage, upper row), HR (middle row) and occurrence of apnea (lower row) analyzed with respect to frequency (left column), amplitude (middle column) and pulse width (right column). Surprisingly, under Metoprolol treatment, sVNS does not potentiate the bradycardic effect of the $\beta$-blocker, whereas the occurrence of apnea increases significantly under Metoprolol treatment. Note, that on average, a $1 \%$ drop in BP is equivalent to a drop of $0.95 \mathrm{~mm}$ Hg (MAP) in the control and a $0.75 \mathrm{~mm} \mathrm{Hg}$ drop (MAP) in the Metoprolol scenario. A full color version of this figure is available at Hypertension Research online.

The frequency had the smallest impact on the BP reduction, without a significant difference within the control group and Metoprolol-treated animals. However, on average in the control animals, the strongest reduction was found at $40 \mathrm{~Hz}$, whereas in
Metoprolol-treated animals the strongest reduction was observed at $50 \mathrm{~Hz}$.

The stimulation amplitude had a strong effect on the BP decrease in both, the control and the Metoprolol-treated animals. With increasing 
stimulation-amplitude ranges (first range: $0.3-0.5 \mathrm{~mA}$, second range: $0.6-1 \mathrm{~mA}$, third range: $1.1-1.3 \mathrm{~mA}$, fourth range: $1.4-1.8 \mathrm{~mA}$, see Figure 5 second column), the BP reduction increased as well, but showed a saturation effect. In the control animals, each increase of the stimulation range (for example, from range two to three) resulted in a significantly bigger BP reduction. However, with each increase of stimulation-amplitude range the P-levels of the Dunn's test statistics became weaker (BP reduction at range one was significantly smaller than in range two at $P<0.001, \mathrm{BP}$ reduction in range two was significantly smaller than in range three at $P<0.001$, BP reduction in range three was significantly smaller than in range four at $P<0.05$, Dunn's test). For the Metoprolol-treated animals, we also found a constant increase in BP reduction with increasing amplitude, but the saturation occurred earlier and the increase became non-significant (range one $<$ range two significant at $P<0.05$, range two $<$ range three non- significant, range $3<$ range 4 non-significant, Dunn's test).

The pulse width also showed a strong yet less homogenous effect on $\mathrm{BP}$ reduction. For the control group, the BP reduction significantly increased, with a broader pulse width up to $0.5 \mathrm{~ms}$ (BP reduction in pulse width range $0.1-0.3 \mathrm{~ms}<$ pulse width range $0.4-0.5 \mathrm{~ms}$ significant at $P<0.0001)$. Higher pulse widths did not result in significantly higher BP decreases. The second smallest BP decrease was found in the pulse width range four from 0.9 to $1.1 \mathrm{~ms}$. The correlation between pulse width and BP reduction was different in the Metoprolol-treated animals. The BP reduction continuously increased with broadened pulse width (with the exception of pulse width range two at $0.4-0.5 \mathrm{~ms}$ ), but this increase did not always reach significant levels (range $1>$ range 2 non-significant, range $2<$ range 3 significant with $P<0.0001$, range $3<$ range 4 non-significant, range $4<$ range 5 significant with $P<0.001$ ).

\section{Response of HR to electrical stimulation before and after} administration of Metoprolol

Before Metoprolol administration, the stimulation-related reduction of HR was influenced by the stimulation frequency, with the maximum observed at $50 \mathrm{~Hz}$ (Figure 5, middle row).

The stimulation amplitude had an even higher impact on the HR reduction, (that is, the higher the amplitude, the stronger the HR reduction), and to a much higher degree than that observed for BP. However, the strongest as well as the most heterogeneous effect on HR was triggered by the stimulation pulse width. Across all stimulation parameters, the highest means for bradycardia were released by stimulations with a pulse width between 0.6 and $0.8 \mathrm{~ms}$.

Under Metoprolol, we did not see any significant bradycardia independent from stimulation frequency, amplitude and pulse width. The highest means in bradycardia across all stimulation parameters were found at pulse widths between 1.2 and $1.8 \mathrm{~ms}$.

Table $1 \mathrm{PQ}$-intervals

\begin{tabular}{lcccc}
\hline & Pre stimulus & Post stimulus & Metoprolol & Post stimulus \\
$(n=10)$ & $(A)$ & $(B)$ & $(C)$ & $M(D)$ \\
\hline Mean & $50 \mathrm{~ms}$ & $48 \mathrm{~ms}$ & $59 \mathrm{~ms}$ & $61 \mathrm{~ms}$ \\
s.d. & $5 \mathrm{~ms}$ & $4 \mathrm{~ms}$ & $10.6 \mathrm{~ms}$ & $5.8 \mathrm{~ms}$ \\
Normal & No & No & No & No \\
distribution & & & & \\
\hline
\end{tabular}

\section{Response of RR to electrical stimulation before and after} administration of Metoprolol

We assessed the occurrence of apnea at any stimulation. With the given RR and the stimulation duration between 3.3 and $2 \mathrm{~s}$, we did not expect to see more than two respiratory dropouts. When comparing the concurrencies of apnea of the control vs the Metoprolol group, it becomes obvious that Metoprolol acts as a strong apnea promoter. In the control stimulation, the highest apnea encounter (of 12\%) was observed in the amplitude stimulation range of 0.6-1 mA. Apnea occurrence never dropped below $15 \%$ at any stimulation condition under Metoprolol, and the highest occurrence (40\%) was found at stimulation pulse width range of 1.2 to $1.8 \mathrm{~ms}$ (Figure 5, lower row). For the control group, the apnea distribution for frequency and amplitude was rather heterogeneous, whereas the apnea risk seemed to drop almost linearly with increasing pulse width.

In the Metoprolol group, the stimulation frequency did not have any influence on RR. Interestingly, there was a slight trend for a higher occurrence of apnea with increasing stimulation frequencies, even though $50 \mathrm{~Hz}$ stimulations were but $2 \mathrm{~s}$ in duration, whereas the $30 \mathrm{~Hz}$ stimulations involved $3.3 \mathrm{~s}$ duration for 100 pulses. The apnea rate with changes in amplitudes resembled, but was the opposite of the occurrence, of apnea found in the control group. Stimulation amplitudes between 0.6 and $1 \mathrm{~ms}$ appeared to be critical to the baroreflex, and this was also the case under Metoprolol.

Response of PQ-intervals to electrical stimulation before and after administration of Metoprolol

The selective stimulation under anesthesia had no significant effect on the PQ-intervals. We found a small and insignificant drop from an average of $50-48 \mathrm{~ms}$ (see Table 1). The application of Metropolol resulted in a highly significant increase of the PQ-time to an average of 59 ms (Dunn's multiple test, A vs $C, P<0.0001$ ). After application of Metoprolol, selective stimulation had a small but significant increase in the PQ-intervals from $59 \mathrm{~ms}$ to an average of $61 \mathrm{~ms}$ (Dunn's multiple test, $\mathrm{C}$ vs $\mathrm{D}, P=0.05$ ).

Finally, the occurrence of apnea showed a reciprocal behavior within different pulse widths. Although the occurrence of apnea decreased with increasing pulse width in the control scenario, it significantly increased with broader pulse widths following the administration of Metoprolol.

As the stimulation frequency was the least important factor for $\mathrm{HR}$ and MAP compared with amplitude and pulse width, Figures 6 and 7 present the BP (Figure 6) and HR (Figure 7) reduction, respectively, in a color-coded contour plot as a function of stimulation pulse width and amplitude. In both figures, the left column shows the control group. Unlike a typical i-t curve (intensity-duration curve), the effect on BP was not continuously elevated with increasing stimulation energy/time (Figure 6). Rather, there seemed to be several 'hot spots' where certain combinations of stimulation parameters reached the highest effectiveness. As shown in the right column, after Metoprolol administration, only a few combinations of amplitude (1.1-1.6 mA) and pulse widths (1.1-1.2) had an effect on BP.

For HR reduction the contour plot for the control group illustrates strong changes between the different stimulation frequencies (Figure 7). At $30 \mathrm{~Hz}$, there did not seem to be any major hot spots for bradycardia except for very high stimulation amplitudes combined with rather short pulse widths. The scenario changed with increasing stimulation frequency, which resulted in the appearance of two large hot spots. Again, the pulse width resulted in the strongest effect at rather low rates. 

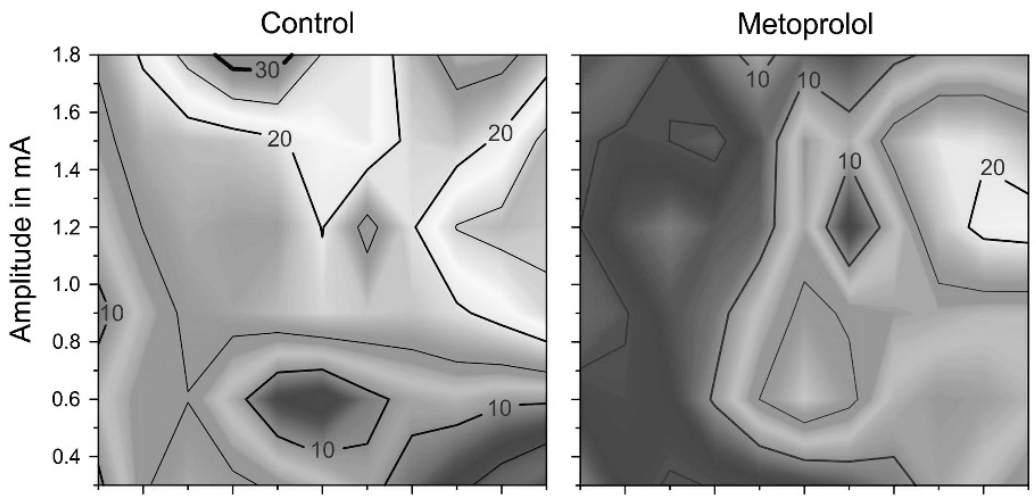

$30 \mathrm{~Hz}$
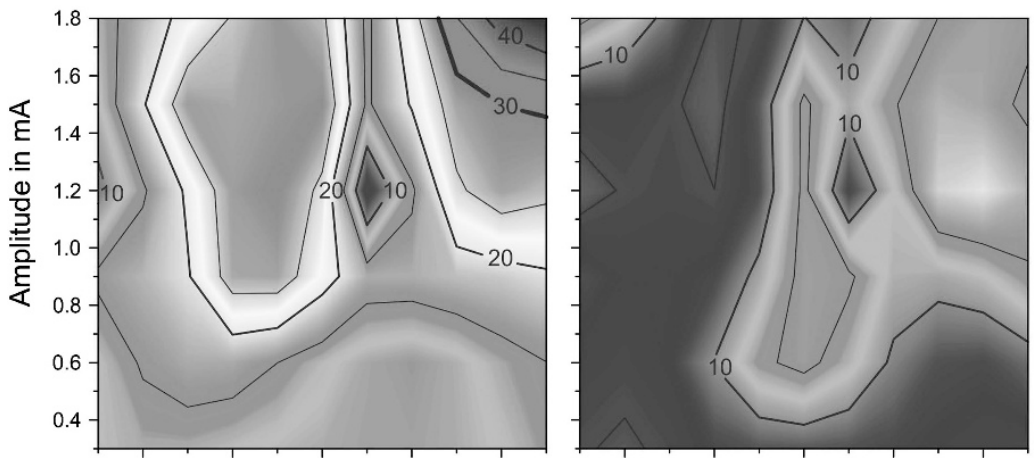

$40 \mathrm{~Hz}$
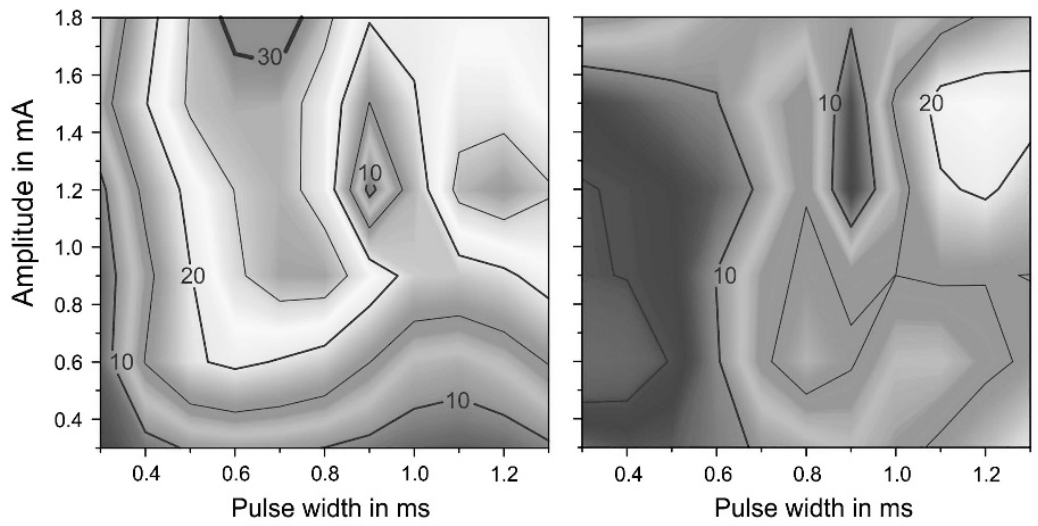

$50 \mathrm{~Hz}$

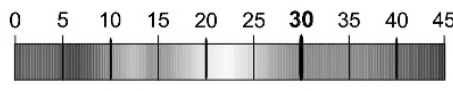

Decrease of BP in \%MAP

Figure 6 Bivariate distribution of the BP decrease as a contour plot. BP reduction is presented in a color-coded contour plot as a function of pulse width and amplitude. The plot gives the averaged results across all test animals. The first column illustrates the BP reduction for the three different stimulation frequencies. Unlike a typical i-t curve (intensity-duration curve), the effect was not elevated with increasing stimulation energy/time. Rather, there seemed to be 'hot spots', where certain combinations of stimulation parameters reached highest effectiveness. After the administration of Metoprolol, only a few combinations of amplitude (1.1-1.6 mA) and pulse widths (1.1-1.2) had an effect on BP. A full color version of this figure is available at Hypertension Research online.

For the Metoprolol group, we had to change the scaling of the bradycardia in percentage in Figure 7, because bradycardia was significantly reduced by the $\beta$-antagonist. Regardless, the trend seems to be the same; the higher the stimulation frequency, the higher is the bradycardia rate.

\section{DISCUSSION}

The activation of the central baroreflex through electrical stimulation of the arterial baroreceptors in the carotid sinus has already been subject of research since the 1960s. ${ }^{19-21}$ Today it is consensus that the baroreflex is not a linear system but influenced by several different parameters, ${ }^{22}$ and that the type of stimulation (permanent vs pulsatile) also has a role in baroreflex sensitivity. ${ }^{23}$ This direction of research was abandoned in light of new upcoming antihypertensive drugs and the technical limitations of the electrodes and batteries. In the early 2000s, new goals in the antihypertensive therapy, which were formulated by the $\mathrm{WHO}$ and aiming at achieving an arterial $\mathrm{BP}$ of below $140 \mathrm{~mm} \mathrm{Hg}$, as well as the development of modern electrode technology shifted the focus of research back to non-medical antihypertensive therapy options. ${ }^{24}$ Modern carotid sinus stimulation can reduce the MAP by $30 \mathrm{~mm} \mathrm{Hg} .8$ 

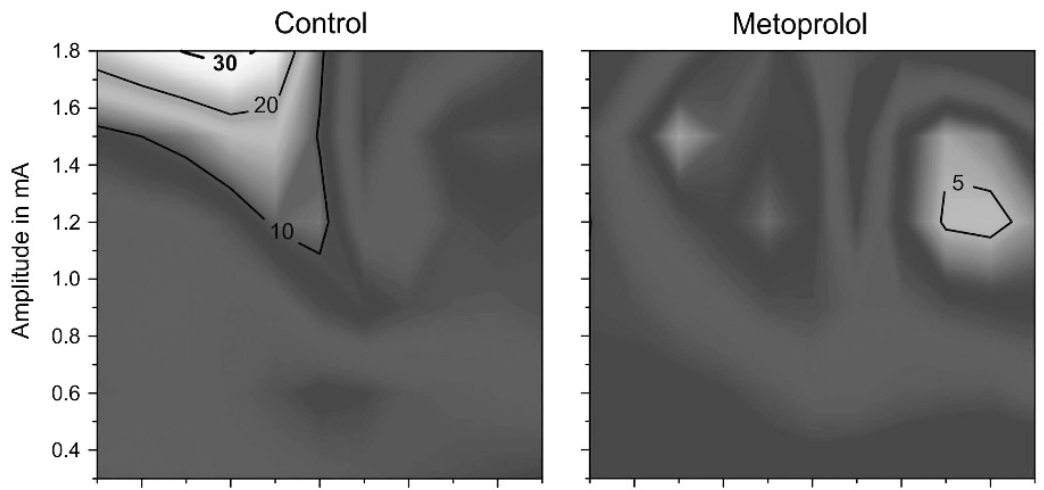

$30 \mathrm{~Hz}$
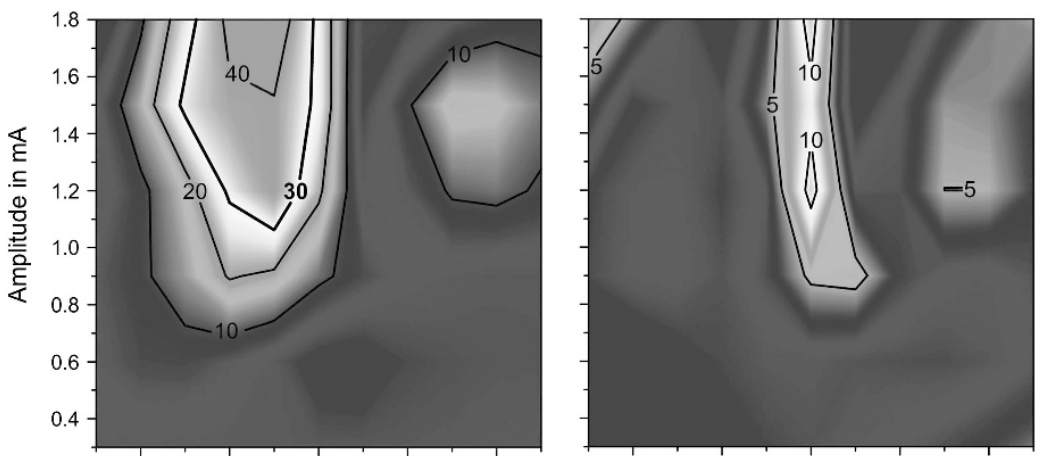

$40 \mathrm{~Hz}$
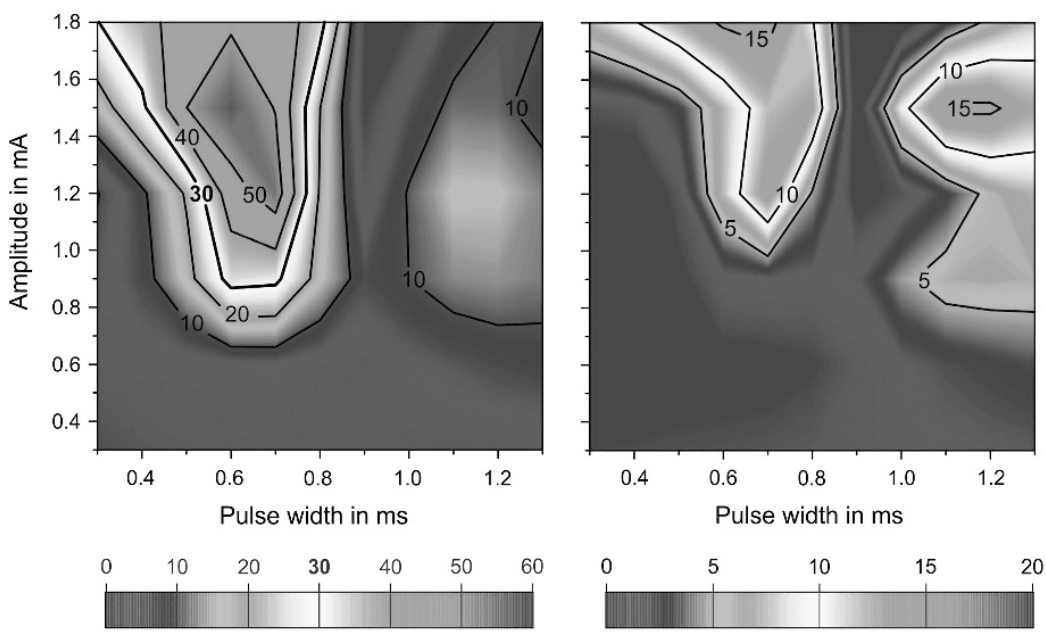

Bradycardia in \%

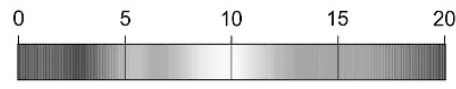

Bradycardia in \%

Figure 7 Bivariate distribution of the HR reduction as a contour plot. HR reduction is presented in a color-coded contour plot as a function of pulse width and amplitude. The plot gives the averaged results across all test animals. For the HR reduction, the contour plot for the control group illustrates strong changes between the different stimulation frequencies. At $30 \mathrm{~Hz}$, there do not seem to be any major hot spots for bradycardia, except for very high stimulation amplitudes at rather short pulse widths. The scenario changed with increasing stimulation frequency, which resulted in the appearance of two large hot spots. Again, the pulse width had the strongest effects at rather low rates. A full color version of this figure is available at Hypertension Research online.

We recently described a method of sVNS for activating the baroreflex for the reduction of BP. ${ }^{14}$ As patients with therapyresistant hypertension treated with sVNS would, owing to their health-related advantages beyond the pure reduction of BP, still keep their basic antihypertensive therapy, it was the aim of this study to investigate how the most commonly used $\beta 1$-selective drug Metoprolol interferes with sVNS in a rat model. It was of significant interest whether the bradycardic effects of $\beta$-blockers and sVNS superimpose and cause a severe bradycardia during stimulation.
Previous studies have demonstrated that the MAP of normotensive, conscious rats is not significantly influenced by the administration of the $\beta 1$-selective hydrophilic agent Atenolol, whereas the HR showed a significant decrease. ${ }^{25-27}$ Activation of the baroreflex through bipolar stimulation of the dissected ADN before medication resulted in a combination of BP reduction and reflex bradycardia. After administration of Atenolol, the effect of ADN stimulation was unaltered, whereas the reflex bradycardia was absent. The studies also demonstrated that spontaneously hypertensive rats showed a much 
higher drop of MAP following both the administration of Atenolol and stimulation of the ADN. ${ }^{28}$ This was explained by the idea that in SHR rats the sympathetic tone is the strongest cause of the elevated MAP and that its blockade with Atenolol results in a distinct drop in MAP.

Consistent with these results, our study in anesthetized rats showed an amplitude-dependent drop of MAP during sVNS before the administration of Metoprolol, with the pulse width being the second strongest, yet most heterogenous parameter. As observed in Figure 6 and Figure 7, only certain combinations of amplitude, pulse width and frequency showed the strongest effects on BP and HR. This result indicates that the baroreflex is far more than a linearly working throttle for adjusting BP and HR, but is also a multifactorial control system that is sensitive to certain combinations of amplitude, frequency and pulse width.

Following Metoprolol treatment, both HR and MAP showed a significant decrease until a steady state was reached after several minutes. Consecutive sVNS reduced both MAP and HR, but this was significantly less pronounced then before the administration of Metoprolol. In particular, the HR reduction was almost eliminated by the application of Metoprolol. This observation might be explained by the hypothesis that with a $\beta$-blocking agent all $\beta$-receptors are already blocked so that the reflex bradycardia cannot be activated by the decreased sympathetic tone of the baroreflex. In addition, Wang et al. ${ }^{29}$ described $\beta$-receptors in the hypothalamic paraventricular nucleus that might also be able to modulate the baroreflex. ${ }^{29}$ Further studies on the influence of these receptors on the baroreflex in the presence of different $\beta$-blocking agents might be interesting.

We also interpret the absence of a severe bradycardia during sVNS and Metoprolol as a demonstration of the selectivity of sVNS, as no efferent cardiac fibers were accidentally stimulated that would still have caused a further reduction of HR in $\beta$-blocked rats. The results of our study are consistent with the results of the aforementioned publications, so we can conclude that sVNS by a cuff electrode wrapped around the vagal nerve bundle stimulates the ADN with a comparable selectivity as stimulation of the dissected ADN does.

The observation that Atenolol did not cause any influence on the baseline MAP and the MAP during ADN stimulation is indeed contrary to our results with Metoprolol. The only noticeable effect of Atenolol was a decrease in baseline HR and a blunted baroreflex, with a less-pronounced bradycardia during ADN stimulation. This difference between our study and the previous work with Atenolol might be caused by the fact that the aforementioned studies were performed in conscious rats, whereas we worked with anesthetized animals. Elevated sympathetic activity in the conscious animals might have blurred the effect that we experienced in the unconscious rats.

Similar to our findings ${ }^{14}$ selective stimulation had hardly any effect on the PQ-intervals. As expected, Metoprolol application significantly elevated the PQ-interval. Under Metoprolol condition, selective vagal stimulation increased the PQ-intervals by an average of $3.3 \%$. However, this finding just stretches into significance with a nivea of $P=0.049$. This observation again indicates that sVNS selectively stimulated the ADN and that efferent stimulation of cardiac fibers was obviously negligible during sVNS.

It has to be mentioned that with the described acute experimental setting we are only able to investigate the acute cardiovascular onset dynamic during sVNS, as we limited the number of pulses to 100 . It is essential to investigate sVNS also in a chronic setting in which the stimulation parameters can be adjusted to reach a cardiovascular steady state. Such a chronic setting will allow to investigate the longterm influence of Metoprolol and other antihypertensive agents in a balanced cardiovascular system. Our group will therefore continue investigating sVNS in normotensive and hypertensive sheep in both acute and chronic-conscious conditions.

We also noticed a significant increase in the incidence of apnea during sVNS after the administration of Metoprolol. To our knowledge, there is no published literature that has investigated the spontaneous RR of rats under sVNS and $\beta$-blockade, and the existing literature has only focused on rats under controlled ventilation. In 1985, Langmeijer et al. ${ }^{30}$ found that overdosing $\beta$-blocking agents resulted in lethal respiratory arrest and found this to owing to impairments in the central respiration control. ${ }^{31}$ It is noticeable that the effect of intravenously administered Propranolol, a very lipophilic $\beta$-blocker, ${ }^{32}$ seemed to be the most prominent compared with other, less lipophilic $\beta$-blockers in that study. Our observation of a massive and highly significant increase in the occurrence of apnea owing to sVNS after administration of Metoprolol suggests that both the baroreflex and Metoprolol have a significant influence on central respiratory control mechanisms. Saku et al. ${ }^{33}$ reported that apnea appears during high-intensity afferent VNS. In contrast to our work, they used only a stimulation frequency of $20 \mathrm{~Hz}$ and a pulse width of $0.2 \mathrm{~ms}$. In our study the occurrence of apnea did not correlate with the intensity of the stimulation, but with their frequency and their pulse width — and only after administration of Metoprolol. Further studies should elucidate whether side effects like apnea could be avoided by chosing the right combination of amplitude, pulse width and frequency instead of stating that apnea only occurs within high stimulation intensities.

\section{CONCLUSION}

sVNS can trigger the baroreflex in rats in an amplitude- and pulse width-dependent manner. Metoprolol reduces HR and MAP and blunts the effects of the baroreflex on both HR and MAP, and therefore did not act as a promotor for severe bradycardia that could have been expected if the baroreflex-induced withdrawel of sympathetic activation and $\beta$-blockade superimposed. It can therefore be assumed that sVNS could possibly used together with $\beta$-blockers without an elevated risk of bradycardia in case sVNS becomes available as an antihypertensive treatment option some day. Respiratory side effects might be avoided by selecting the correct set of parameters especially with respect to stimulation frequency and pulse width, as these side effects might correlate less with stimulation amplitude then expected.

\section{CONFLICT OF INTEREST}

The authors declare no conflict of interest.

\section{ACKNOWLEDGEMENTS}

We thank Oscar Cota, Fabian Boeser, Thomas Stieglitz and Josef Zentner for their help. This work was supported by the B Braun foundation and the Research Foundation of the German Neurosurgical Society DGNC.

\section{DISCLAIMER}

The funding foundations were not involved in the study design, collection, analysis, interpretation, writing or decision-making.

1 Cutler JA, Sorlie PD, Wolz M, Thom T, Fields LE, Roccella EJ. Trends in hypertension prevalence, awareness, treatment, and control rates in United States adults between 1988-1994 and 1999-2004. Hypertension 2008; 52: 818-827.

2 Whitworth JA. World Health Organization; International Society of Hypertension Writing Group 2003 World Health Organization (WHO)/International Society of Hypertension (ISH) statement on management of hypertension. J Hypertens 2003; 21: 1983-1992. 
3 Tomiyama H, Yoshida M, Higashi Y, Takase B, Furumoto T, Kario K, Ohya Y, Yamashina A. Sub-group study of FMD-J. Autonomic nervous activation triggered during induction of reactive hyperemia exerts a greater influence on the measured reactive hyperemia index by peripheral arterial tonometry than on flow-mediated vasodilatation of the brachial artery in patients with hypertension. Hypertens Res 2014; 37: 914-918.

4 Chobanian AV, Bakris GL, Black HR, Cushman WC, Green LA, Izzo JL Jr., Jones DW, Materson BJ, Oparil S, Wright JT Jr., Roccella EJ. Joint National Committee on Prevention, Detection, Evaluation, and Treatment of High Blood Pressure; Nationa Heart, Lung, and Blood Institute; National High Blood Pressure Education Program Coordinating Committee Seventh report of the Joint National Committee on Prevention, Detection, Evaluation, and Treatment of High Blood Pressure. Hypertension 2003; 42: 1206-1252.

5 Grassi G, Bombelli M, Buzzi S, Volpe M, Brambilla G. Neuroadrenergic disarray in pseudo-resistant and resistant hypertension. Hypertens Res 2014; 37: 479-483.

6 Scheffers IJM, Kroon AA, Tordoir JHM, de Leeuw PW. Rheos Baroreflex Hypertension Therapy System to treat resistant hypertension. Expert Rev Med Devices 2008; 5: 33-39.

7 Heusser K, Tank J, Engeli S, Diedrich A, Menne J, Eckert S, Peters T, Sweep FCGJ, Haller H, Pichlmaier AM, Luft FC, Jordan J. Carotid baroreceptor stimulation, sympathetic activity, baroreflex function, and blood pressure in hypertensive patients. Hypertension 2010; 55: 619-626.

8 Bisognano JD, Bakris G, Nadim MK, Sanchez L, Kroon AA, Schafer J, de Leeuw PW, Sica DA. Baroreflex activation therapy lowers blood pressure in patients with resistant hypertension: results from the double-blind, randomized, placebo-controlled rheos pivotal trial. J Am Coll Cardiol 2011; 58: 765-773.

9 Benarroch EE. The arterial baroreflex: functional organization and involvement in neurologic disease. Neurology 2008; 71: 1733-1738.

10 Dworkin BR, Dworkin S, Tang X. Carotid and aortic baroreflexes of the rat: I. Open-loop steady-state properties and blood pressure variability. Am J Physiol Regul Integr Comp Physiol 2000; 279: R1910-R1921.

11 Ramani R. Vagus nerve stimulation therapy for seizures. J Neurosurg Anesthesiol 2008; 20: 29-35.

12 Beekwilder JP, Beems T. Overview of the clinical applications of vagus nerve stimulation. J Clin Neurophysiol 2010; 27: 130-138.

13 Plachta DT, Gierthmuehlen M, Cota O, Boeser F, Stieglitz T. BaroLoop: using a multichannel cuff electrode and selective stimulation to reduce blood pressure. Conf Proc IEEE Eng Med Biol Soc 2013, 2013: 755-758.

14 Plachta DT, Gierthmuehlen M, Cota O, Espinosa N, Boeser F, Herrera TC, Stieglitz T, Zentner J. Blood pressure control with selective vagal nerve stimulation and minimal side effects. J Neural Eng 2014; 11: 036011.

15 Klimas J, Kruzliak P, Rabkin SW. Modulation of the QT interval duration in hypertension with antihypertensive treatment. Hypertens Res 2015; 38: 447-454.

16 Plachta DTT, Gierthmuehlen M, Stieglitz T. Selective stimulation of the vagal nerve reduces blood pressure without side effects. Biomed Tech 2012; 57 (Suppl. 1) DOI 10.1515/bmt-2012-4289.
17 Zariffa J, Popovic MR. Influence of the number and location of recording contacts on the selectivity of a nerve cuff electrode. IEEE Trans Neural Syst Rehabil Eng 2009; 17: 420-427.

18 Waynforth HB, Flecknell PA. Experimental and Surgical Technique in the Rat. Academic Press, New York, 1992.

19 Bilgutay A, Lillehei C. Treatment of hypertension with an implantable electronic device. JAMA 1965; 191: 649-653.

20 Bilgutay AM, Lillehei CW. Surgical treatment of hypertension with reference to baropacing. Am J Cardiol 1966; 17: 663-667.

21 Neistadt A, Schwartz S. Effects of electrical stimulation of the carotid sinus nerve in reversal of experimentally induced hypertension. Surgery 1967; 61: 923-931.

22 Schreihofer AM, Mandel DA, Mobley SC, Stepp DW. Impairment of sympathetic baroreceptor reflexes in obese Zucker rats. Am J Physiol 2007; 293: H2543-H2549.

23 Chapleau MW, Abboud FM. Contrasting effects of static and pulsatile pressure on carotid baroreceptor activity in dogs. Circ Res 1987; 61: 648-658.

24 Mohaupt MG, Schmidli J, Luft FC. Management of uncontrollable hypertension with a carotid sinus stimulation device. Hypertension 2007; 50: 825-828.

25 Salgado H, Barale A, Castania J, Machado B, Chapleau M, Fazan R Jr. Baroreflex responses to electrical stimulation of aortic depressor nerve in conscious SHR. Am J Physiol 2007; 292: 593-600.

26 Japundzic N, Grichois ML, Zitoun P, Laude D, Elghozi JL. Spectral analysis of blood pressure and heart rate in conscious rats: effects of autonomic blockers. J Auton Nerv Syst 1990; 30: 91-100.

27 Murphy CA, Sloan RP, Myers MM. Pharmacologic responses and spectral analyses of spontaneous fluctuations in heart rate and blood pressure in SHR rats. J Auton Nerv Syst 1991; 36: 237-250.

28 Durand Mde T, Castania JA, Fazan R Jr., Salgado MC, Salgado HC. Hemodynamic responses to aortic depressor nerve stimulation in conscious L-NAME-induced hypertensive rats. Am J Physiol Regul Integr Comp Physiol 2011; 300: R418-R427.

29 Wang D, Feng H, Li YS, Qiu DL, Jin H, Jin QH. Beta-adrenoceptors in the hypothalamic paraventricular nucleus modulate the baroreflex in conscious rats. Neurosci Lett 2013; 551: 43-46.

30 Langemeijer J, de Wildt D, de Groot G, Sangster B. Respiratory arrest as main determinant of toxicity due to overdose with different beta-blockers in rats. Acta Pharmacol Toxicol 1985; 57: 352-356.

31 Langemeijer JJ, de Wildt DJ, de Groot G, Sangster B. Central origin of respiratory arrest in beta-blocker intoxication in rats. Toxicol Appl Pharmacol 1987; 89: 399-407.

32 Dreisbach AW, Greif RL, Lorenzo BJ, Reidenberg MM. Lipophilic beta-blockers inhibit rat skeletal muscle mitochondrial respiration. Pharmacology 1993; 47: 295-299.

33 Saku K, Kishi T, Sakamoto K, Hosokawa K, Sakamoto T, Murayama Y, Kakino T, Ikeda M, Ide T, Sunagawa K. Afferent vagal nerve stimulation resets baroreflex neural arc and inhibits sympathetic nerve activity. Physiol Rep 2014; 2: e12136. 